\title{
Development of Personal Wellness Information Model for Pervasive Healthcare
}

\author{
Antto Seppälä, ${ }^{1}$ Pirkko Nykänen, ${ }^{1}$ and Pekka Ruotsalainen ${ }^{2}$ \\ ${ }^{1}$ eHealth Research Group, School of Information Sciences, University of Tampere, P.O. BOX 607, 33014 University of Tampere, Finland \\ ${ }^{2}$ Department of Information, National Institute for Health and Welfare, P.O. Box 30, 00271 Helsinki, Finland
}

Correspondence should be addressed to Antto Seppälä, antto.seppala@uta.fi

Received 2 March 2012; Accepted 8 August 2012

Academic Editor: Rui Zhang

Copyright (C) 2012 Antto Seppälä et al. This is an open access article distributed under the Creative Commons Attribution License, which permits unrestricted use, distribution, and reproduction in any medium, provided the original work is properly cited.

\begin{abstract}
Pervasive healthcare and citizen-centered care paradigm are moving the healthcare outside the hospital environment. Healthcare delivery is becoming more personalized and decentralized, focusing on prevention and proactive services with a complete view of health and wellbeing. The concept of wellness has been used to describe this holistic view of health, which focuses on physical, social, and mental well-being. Pervasive computing makes it possible to collect information and offer services anytime and anywhere. To support pervasive healthcare with wellness approaches, semantic interoperability is needed between all actors and information sources in the ecosystem. This study focuses on the domain of personal wellness and analyzes related concepts, relationships, and environments. As a result of this study, we have created an information model that focuses on the citizens' perspectives and conceptualizations of personal wellness. The model has been created based on empirical research conducted with focus groups.
\end{abstract}

\section{Introduction}

Healthcare delivery is undergoing a notable shift toward personalized services with distributed care processes that emphasize a more holistic view of health and wellness within a citizen-centered care model [1-6]. The new citizencentered care paradigm focuses on the health, functioning, and well-being of people as a whole [7]. The focus, then, is more on preventive, proactive services with the citizen at the core of his/her care, instead of just treating diseases and symptoms $[1,2,4-6]$. In the future, it will not be enough to just access medical histories or test results, but citizens' lifestyle information, behavioral choices, and monitoring and measurement data will need to be considered to ensure preventive, proactive service $[2,4-6]$.

Such a view of health is not entirely new. The World Health Organization (WHO) defined health as early as 1948 as "a state of complete physical, mental and social well-being and not merely the absence of disease of infirmity" [8, page 100]. This definition acknowledged a holistic view of health and supported a focus beyond disease treatment. Despite this WHO's definition, the concept of health may cause misunderstandings because usually it is understood to refer to a person's state when free of diseases, and the focus is on medical well-being. However, health can be defined in many ways, for example, it can be seen as a state of stable physiological function, lack of diseases, and absence of illnesses. One term that has been used to describe more complete health and well-being of people is wellness. Wellness is thought to be a subjective feeling of health and well-being [9]. Wellness considers individuals' general functioning as a whole, covering not only physical but also social and psychological aspects $[9,10]$. Wellness is a multidimensional concept, and it is currently being studied in many different scientific areas, such as medicine, public health, occupational health, and mental health [9].

\section{Research Context and Objectives}

Pervasive healthcare can be defined either as the application of pervasive computing -in other words, ubiquitous computing, proactive computing, or ambient intelligencefor healthcare, health, and wellness management, or as making healthcare available anytime and anywhere [11]. 
The basic idea behind pervasive healthcare is to integrate healthcare technologies and concepts into people's everyday lives. Pervasive healthcare is seen as supporting the shift toward citizen-centered care $[12,13]$. Very closely related to pervasive healthcare is the research being done in the pervasive and ubiquitous computing fields. These technology paradigms focus on embedded, mobile, proactive, contextaware, collaborative, and sensor systems [13].

Pervasive healthcare applications are designed to support decentralized and preventive care. Pervasive healthcare will also help citizens manage their personal health and wellness outside the provider network. This model highlights a wellness-centered approach by helping citizens to stay well physically, mentally, and socially and to utilize different selfmanagement and assistive services [13]. With pervasive computing technologies, it is it possible to collect all kinds of data anytime and anywhere $[11,14]$. Data collection can be done by using intelligent sensors and measurement technologies.

The objective of information modeling is to describe the information in a certain domain or in an organization. Information models can be used to capture users' perceptions and understanding of system complexities [15]. In this study, we are focusing on the conceptual level of modeling. Conceptual modeling takes as its point of interest knowledge about the domain, and the idea is to build a representation of the real-world semantics $[16,17]$. Conceptual modeling can be performed using informal or semiformal models. Despite of the formality level of used modeling notation, the main aim is to capture concepts and relationships in the target domain $[18,19]$.

With pervasive healthcare and more personalized, holistic, and citizen-centered services, we need a better understanding of the personal wellness domain. This requires more information than typical health records include. To fully support the new ways of managing health and wellness with ICT and pervasive computing, we need to achieve semantic interoperability among different stakeholders, systems, devices, sensors, and other information sources and users. To achieve semantic interoperability, a context-aware personal wellness ontology is needed. This ontology can be used to create shared understandings and allow for sharing of heterogeneous information among all actors and systems in the personal wellness ecosystem.

This study is a part of a research project that examines the trusted use of personal health and wellness information in future ubiquitous computing environments [20]. In this research project, we aim to create a trusted context-aware ontology for lifelong personal wellness management and an architecture model for trusted use of multisource heterogeneous information. Before we can start building a personal wellness ontology, we have to understand what wellness is, what its components are, what information is related to it, how people manage and maintain wellness, and what influences wellness. Basically, we have to uncover the scope and contents of the concept of wellness. This study develops the wellness concept further and builds the basis for the development of a personal wellness ontology.

The first research objective of this study was to analyze the domain of personal wellness. This includes both how the concept of personal wellness is defined in the literature and how people conceptualize it as well as the scope of the domain. The second objective of this study was to create a high-level personal wellness information model to present related concepts, characteristics, and contextual aspects. With this high-level information model, we can understand the concept of personal wellness and start to create a more formal model, in other words, a personal wellness ontology.

\section{Methods}

As a guiding framework for our research, we have used a design science research (DSR) approach $[21,22]$. DSR can be seen as a problem-solving process in which actual business needs are addressed by building actual artifacts or applications. DSR is usually considered to address particularly problematic situations that are characterized by unstable requirements and constraints, complex interactions, a tendency to change in terms of design processes or artifacts, and dependence upon human cognitive and social abilities [22].

DSR is based on two design processes: build and evaluate. These two processes produce IT artifacts, which can be constructs, models, methods, or implementations. Usually build and evaluate processes are performed iteratively to improve the quality of the artifact. Hevner et al. [22] have developed seven guidelines for DSR, and we have followed these during our research. These guidelines are (a) design as an artifact, (b) problem relevance, (c) design evaluation, (d) research contribution, (e) research rigor, (f) design as a search process, and $(\mathrm{g})$ communication of research. In this study, we focus on building and evaluating both the constructs and a model in the personal wellness domain. The constructs define the basic concepts, the universe of discourse, and the relations and attributes of the concepts.

Most of the work done in the domain of personal wellness is related to the measurement or assessment of wellness or well-being. They focus on different things than what we need to develop a personal wellness ontology. These models are high-level descriptions with limited analysis of the concepts or relations. Moreover, environmental factors are defined quite narrowly. In our research, we are more interested in identifying and defining main components, concepts, and relations in the personal wellness domain. We are creating a more complete and conceptually focused model to support the field of pervasive healthcare.

This study had three main methods for information modeling:

(1) an analysis of the literature, which explores the conceptualization of personal wellness, personalized healthcare, and a holistic view of health;

(2) a context analysis to identify internal and external contexts of personal wellness;

(3) focus groups to understand how people understand the concept of personal wellness, how it can be conceptualized, and which factors affect personal wellness. 
TABLE 1: Focus group meetings.

\begin{tabular}{llll}
\hline Group & Duration & Participants & Focus \\
\hline Group 1 & $\begin{array}{l}2 \text { meetings, } \\
\text { 4 hours each }\end{array}$ & $\begin{array}{l}\text { Young, healthy department staff members, open voluntary } \\
\text { call to our department's mailing list (5 and 4 participants) }\end{array}$ & $\begin{array}{l}\text { Identify and describe basic concepts of personal } \\
\text { wellness }\end{array}$ \\
\hline Group 2 & $\begin{array}{l}1 \text { meeting, } \\
2 \text { hours }\end{array}$ & Internal project meeting (4 participants) & $\begin{array}{l}\text { Specification of concepts, redundancy reduction, } \\
\text { and abstraction levels }\end{array}$ \\
\hline Group 3 & $\begin{array}{l}1 \text { meeting, } \\
2 \text { hours }\end{array}$ & $\begin{array}{l}\text { Our university's health informatics postgraduate students } \\
\text { (5 participants) }\end{array}$ & $\begin{array}{l}\text { Concept specification and categorization and } \\
\text { external contexts }\end{array}$ \\
\hline Group 4 & $\begin{array}{l}2 \text { meetings, } \\
2 \text { hours each }\end{array}$ & $\begin{array}{l}\text { Females aged 48-62 (9 and 10 participants); group was } \\
\text { formed on a voluntary basis by sending invitations to } \\
\text { participants in another well-being-related study }\end{array}$ & $\begin{array}{l}\text { Discussion about participants' views on personal } \\
\text { wellness in general and then our models in more } \\
\text { detail }\end{array}$ \\
\hline
\end{tabular}

3.1. Literature Analysis. As a basis for the study, we carried out an analysis of scientific literature concerning personal wellness. The analysis included Google Scholar and different scientific publication databases: Association for Computing Machinery (ACM), ESBCOhost Academic Search Premier, IEEE, PubMed, ScienceDirect, and SpringerLink. For more detailed analysis, we chose articles concerning holistic and multidimensional views on health and wellness that possibly were related to the components of a citizen-centered healthcare paradigm. We manually analyzed around 100 articles concerning these topics.

3.2. Context Analysis. The context analysis was performed using basic techniques from the requirements elicitation process. Requirements elicitation is a process used to find necessary requirements for computer-based systems [23]. In our case, the target was the existing knowledge in the literature. We analyzed the application domain, identified the source documents related to our domain, and recognized and analyzed related stakeholders. The stakeholders, in our case, were the internal and external contexts in personal wellness. We identified both external environment and internal environment, the latter of which consists of factors related directly to the person herself, such as things that she herself can affect, control, influence, or manage.

3.3. Empirical Studies with Focus Groups. From the literature and context analysis, we obtained the material that formed the basis of our empirical research, which was performed with focus groups. A focus group is a method for group interviews in which emphasis is on the communication between the participants. The number of groups or participants may vary, but according to Kitzinger [24], the ideal number of participants in the group is from four to eight. The idea is to generate data based on interaction and communication, instead of the researcher asking the participants direct questions. Focus groups are a useful method for exploring knowledge and experiences of people because they can help people explain and clarify their thoughts and views. It is especially suitable for situations in which the existing knowledge is inadequate, the subject is very complex with many variables, and the research questions are very open. Sometimes, though, the group dynamics might silence some participants or ideas. Methods for conducting focus groupcentered research may vary, depending on several factors, such as the number of participants or groups, how the groups are formed (preexisting or unknown), and the homogeneity or heterogeneity of the participants $[24,25]$.

The total number of focus group meetings in our study was six, with four different groups (see Table 1). The focus group meetings were organized such that one of the researchers acted as a head of the meetings and the researchers collected the results based on the discussions. To support the meetings, we had some themes and models, but the structure of the focus groups meetings was quite relaxed and informal, and the participants were encouraged to discuss their views openly. The objective of the focus groups was to gather empirical material about how personal wellness is understood and conceptualized and what kinds of contexts are related to it. This meant that it was important to collect concepts and the ideas behind them that were revealed during the groups' communication.

The results of the first two meetings were documented in a mind map. Mind mapping was chosen because it is an easy way to represent information, simple categorizations, and simple relations between concepts. Moreover, most people are familiar with this technique. Based on the first two focus group meetings and the performed analyses, we started to model the personal wellness domain in a more formal way by using a simplified entity relationship (ER) notation. The idea behind the use of the simplified ER model was that the model could be easily understood and modified by people without any modeling experience. The results and modifications made by the focus group participants were modeled by the researchers. Although we performed the modeling ourselves based on the focus group meetings, we tried, to the extent possible, to keep the model based on the actual discussions, concepts, and views presented by the focus group participants. The modeling work was divided into seven sub-models, which together form the high-level information model of personal wellness. The focus group meetings were organized to support iterative build-evaluate-type process.

\section{Results}

4.1. Conceptualizing Personal Wellness: Results from the Literature and Context Analysis. Wellness is a multidimensional and multidisciplinary concept. Although definitions may vary depending on the context, generally, wellness is thought to be a balanced state of a healthy body, mind, and 


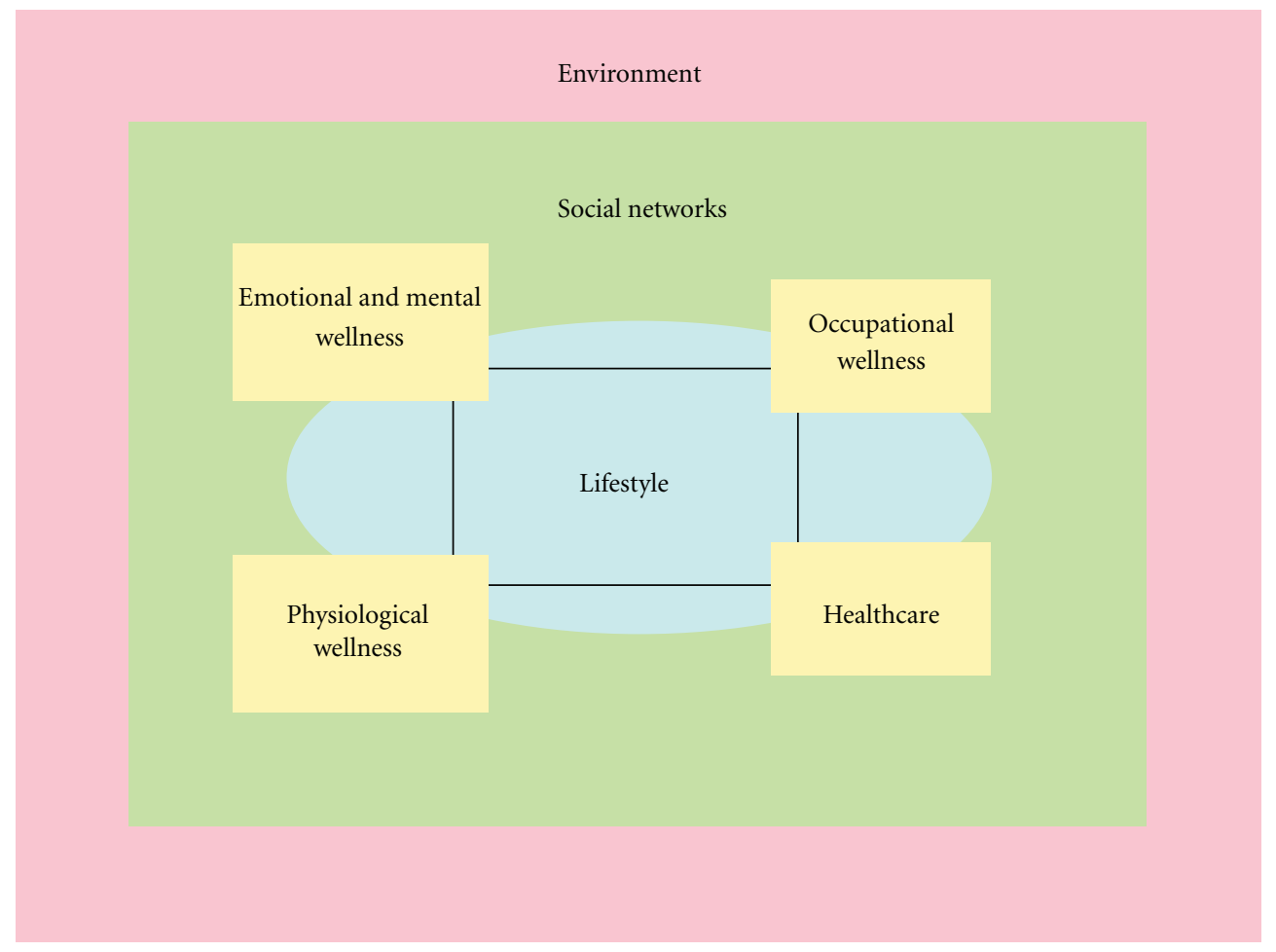

FIgure 1: Personal wellness domain.

spirit [7, 9, 10, 26-28]. Wellness can be seen as a highlevel concept that integrates multiple domains, including the physical, psychological, social, and spiritual domains, and it may vary according to age or cultural context [29]. Also, health promotion, prevention, and better functioning are closely related to wellness $[7,27,30,31]$. Wellness is a complete, holistic view that focuses on the individual and his specific needs. It takes into account the whole person and her environment, acknowledging lifestyle, behavior, culture, beliefs, experiences, and other aspects that affect a person's life $[9,10,27,31-33]$.

Some holistic wellness models have been developed in the field of clinical and counseling psychology [34]: the Wheel of Wellness by Sweeney and Witmer [35], the Indivisible Self by Myers and Sweeney [26], the Circle of Health by Saylor [36], and an ecosystemic approach to health, wellbeing, and wellness by Kirsten et al. [28]. All four models take a holistic, multidimensional view of health and wellness. The models were quite high-level, informal, and general, and their purpose was different from ours, but we were able to extract some of their concepts and other characteristics. For more information about these models and analyses, see [37].

Based on the literature and context analyses, we were able to identify some common concepts, characteristics, and properties of personal wellness. As a result of the analyses, we were able to discover how wellness is defined as a highlevel concept, as well as the common characteristics and components wellness is generally thought to have [37]:

(i) It is a holistic, multidimensional, and multidisciplinary view of health and well-being; (ii) Wellness is a broader concept than most views on health as defined by healthcare; it takes into account environmental, emotional, intellectual, occupational, social, and spiritual aspects of well-being;

(iii) It focuses on complete health and well-being, prevention, and proactive services;

(iv) It is dynamic and context-dependent.

4.2. Empirical Refinement of the Personal Wellness Concept. Based on the literature and context analyses and the first two focus group meetings, we created our own view of personal wellness (Figure 1). The view of personal wellness consists of five internal components and two external contexts. The internal components are: lifestyle, emotional and mental wellness, occupational wellness, healthcare, and physiological wellness. The two external contexts are: social networks and environment. In our view, all components are linked together, which represents the complete and holistic nature of personal wellness in which there are many different relationships between components. This view emphasizes the notion that personal wellness is much more than just physical health and well-being. In this light, it is clear that it should be examined in a multidisciplinary and multi-professional context.

The lifestyle component is a kind of background component that directly affects the other four internal components. The lifestyle component includes concepts regarding activities, behaviors, choices, and risk factors related to person's lifestyle. Emotional and mental wellness focuses on concepts concerning individual identity, psychological concepts, intellectual wellness, emotions, feelings, and so 
forth. Emotional and mental wellness also includes a person's views and values that affect choices and behaviors.

Occupational wellness is about well-being related to people's occupations, and in this context, occupation can mean that the person is working, studying, unemployed, retired, or an entrepreneur. Occupational wellness considers the actual occupation and its effects on personal wellness. Physiological wellness focuses on information related to health and wellness that is not bound to certain healthcare provider, for example, conditions, disabilities, functioning, genetics, monitoring and measuring data, personal observations, and so forth. The healthcare component is about the healthcare system. It includes providers, medical documents, and services.

All of these internal components of personal wellness are surrounded and affected by external contexts-social networks and environment. Social networks include all social relations that affect a person. Environment describes the digital and physical environments related to the person in question. It includes, for example, living environment, service environment, society, cultural aspects, and regulations. The environment surrounding a person is very dynamic, flexible, and sensitive, and it may change over the course of a lifetime.

From the first two focus group meetings alone, we already had quite a lot of different concepts related to personal wellness. We had gathered these concepts into two mind maps, so we had some categorization and simple relations between these concepts. From these mind maps, we started to categorize concepts into models, loosely based on the simplified ER diagram. We created seven different models to represent all the components of personal wellness. In these models, we focused more on the concepts and their categorization because these are core components of ontology development.

We started going through these models with the different focus groups to get a more detailed view and to discover the necessary concepts related to personal wellness. In the focus group meetings, the participants were encouraged to openly discuss current themes. Between the focus group meetings, we analyzed the discussions and the feedback for the meetings and based on this, we revised the models, analyzed and added new concepts into the models, reduced redundant concepts, and considered different relationships between concepts. The resulting high-level personal wellness information model will be introduced in more detail in the next section.

4.3. Personal Wellness Information Model. As a result of the combination of all three methods, we were able to create an information model that describes our view of personal wellness. The lifestyle component describes the domain of personal lifestyle and consists of activities, behaviors, and choices that affect the person's daily life. It also includes active wellness activities and management concepts. These concepts are such that they can usually be affected, controlled, influenced, or managed by the person herself. Lifestyle focuses on concepts that are quite dependent on the person herself, and their emphasis may vary a lot between people. Listed below are the main concepts and subconcepts of lifestyle: (i) livelihood;

(ii) nutrition-experiences, history, food diaries, diet;

(iii) rest/relaxation, sleep;

(iv) risk factors-behavioral, environmental, inheritable;

(v) sexual behavior;

(vi) wellness activities-objectives, self-development, wellness maintenance (personal responsibility, active actions; self-evaluation), hobbies, leisure, fitness/ exercise;

(vii) Wellness behavior-wellness management (activity management, alternative medicine, behavior management, conditions, devices, medication, nonprescribed medication), health education, prevention.

The emotional and mental component focuses on elements related to people's minds, feelings, emotions, identities, and personalities (Figure 2). The concepts in emotional and mental wellness describe personal views, feelings, and attitudes towards life, personality, and experiences. They also include mental health and possible disorders. This component is aimed at describing what is going on in the person's mind, how he will react to different situations, and how he can cope mentally. In the emotional and mental component, we used the categorization of Emotion Annotation and Representation Language (EARL) [38] to describe the emotions of a person.

Occupational wellness describes well-being related to person's occupation. A person's occupation can be, for example, work, studying, or being unemployed or retired. The concept of occupation describes the actual occupation, its properties, or how the person feels about it. It has some subconcepts, which include: appreciation, benefits, equality, functioning, meaningful, motivation, performance, responsibilities, respect, rewarding, rights, and type. Occupational wellness includes also following concepts: education, employer, environment, income, occupational safety and health, quality of working life, social relations, vacation, volunteering, work burden, work climate, work culture (including leadership and management), working ability, and working time.

Physiological wellness has to do with health and wellnessrelated information outside the healthcare provider network. It will help the citizen to collect, observe, and manage her personal health information. It will include different health and wellness devices and their monitoring and measuring of data as well as information about a person's conditions and functioning. Physiological wellness is closely related to the concept of the personal health record, but the idea is to be more thorough and complete through an emphasis on information and events outside the healthcare provider network. It includes the following concepts: conditions, demographic information, disability, functioning, genetic information, measurement and monitoring data, observation, organ system, vital signs, and wellness devices.

The healthcare component focuses on the clinical side of wellness. It has to do with the regulated healthcare system 


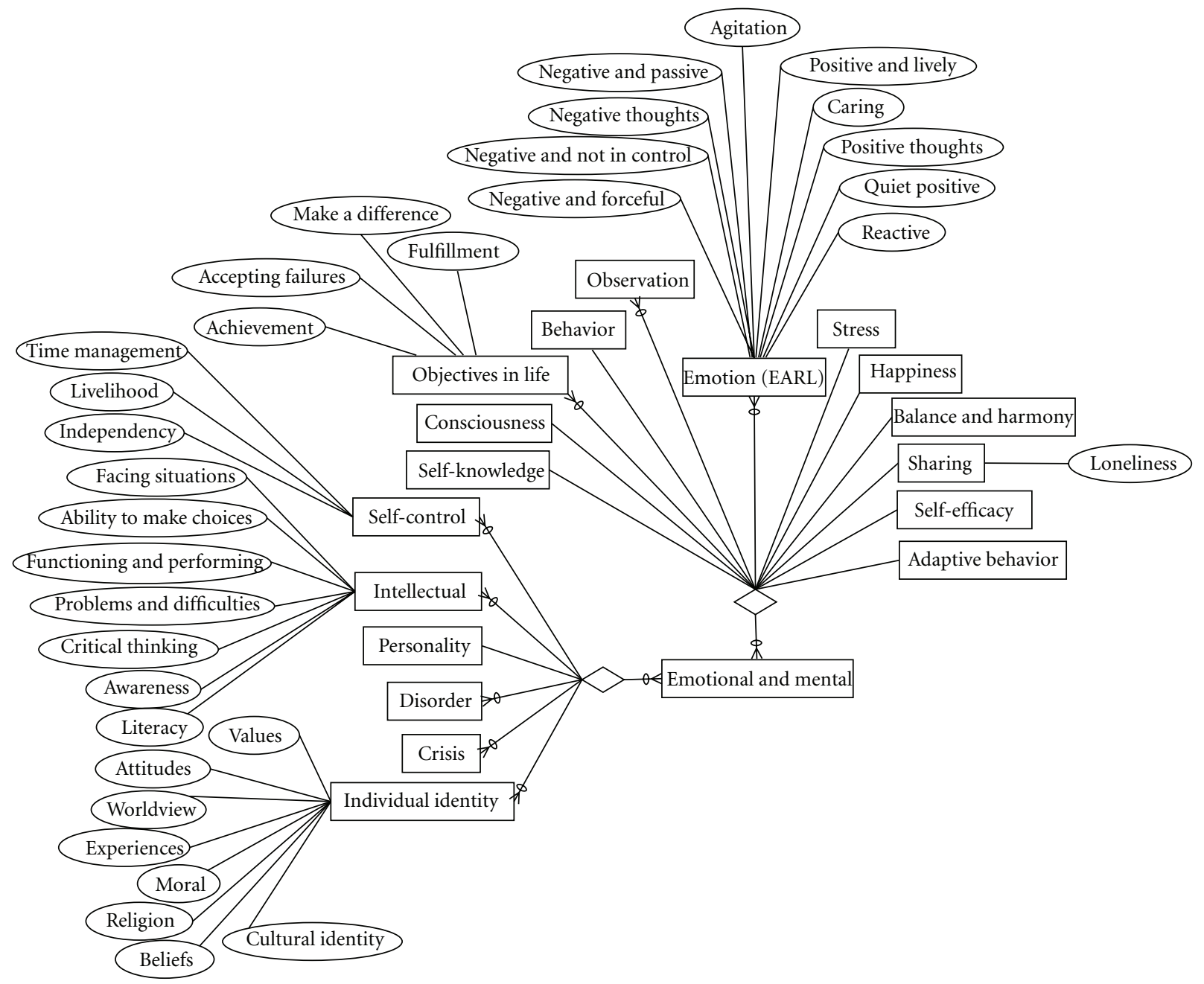

FIGURE 2: Emotional and mental wellness.

and the actual care that the person receives. The model consists of two main concepts: service and provider. The service concept is divided into three subconcepts, or types: prevention, diagnosis, and treatment. All of these different services create medical documents, and from these documents, the medical history of a person is created. Providers are divided into four subconcepts, or types: preventive, curative, promotional, and rehabilitative.

The social networks component describes the different social relations and networks that are related to a person. It includes family, relatives, and friends, but also different communities and social environments, and the social participation of a person within these contexts. Most of the external contexts that affect people's living and personal wellness are located in the environment component. This includes different kinds of environments, such as society, cultural norms, or the media (Figure 3). The environment affects the person in many ways, and it may shape the possibilities for handling personal wellness. These external factors are heavily context-dependent and may differ from country to country.
All seven components combined create a high-level information model of the personal wellness domain (Figure 4).

\section{Discussion}

As a result of this research, we were able to create a high-level information model of the personal wellness domain. Usually, health-related information models and ontologies are built from the healthcare systems and the service providers' viewpoint. Here, however, we have instead created our model from the citizens' viewpoint. The citizens' viewpoint is based on the empirical research performed with the focus groups. We tried to get a comprehensive view with participants from different age groups with different backgrounds but as the focus groups were created on a voluntary basis from a limited pool of people there can be some limitations in the model. Also the participants can be seen being more interested in their health and wellness than regular citizens as they are willing to participate in this kind of voluntary research. With focus groups we have created a model based on active 


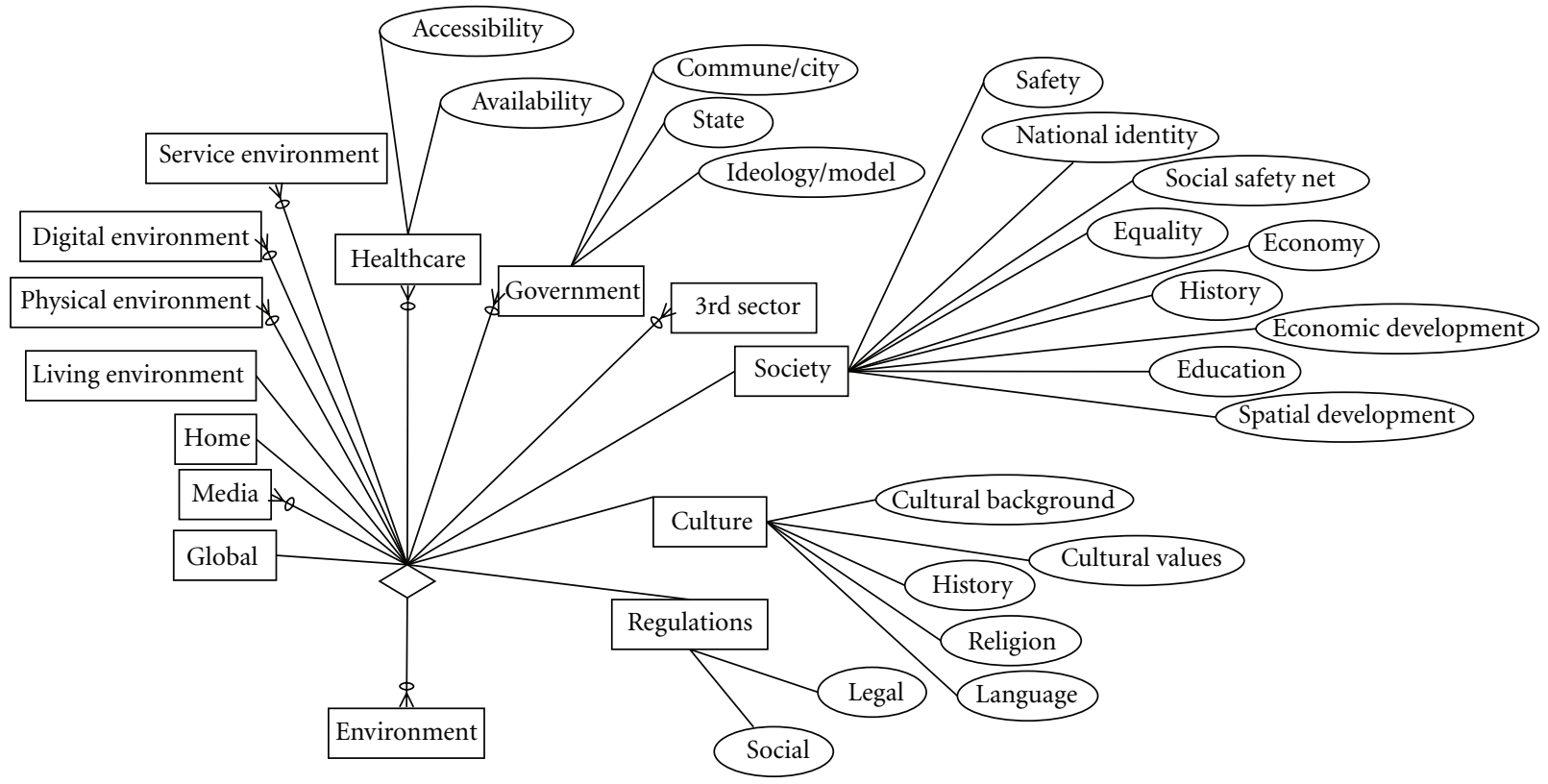

Figure 3: The environment component.

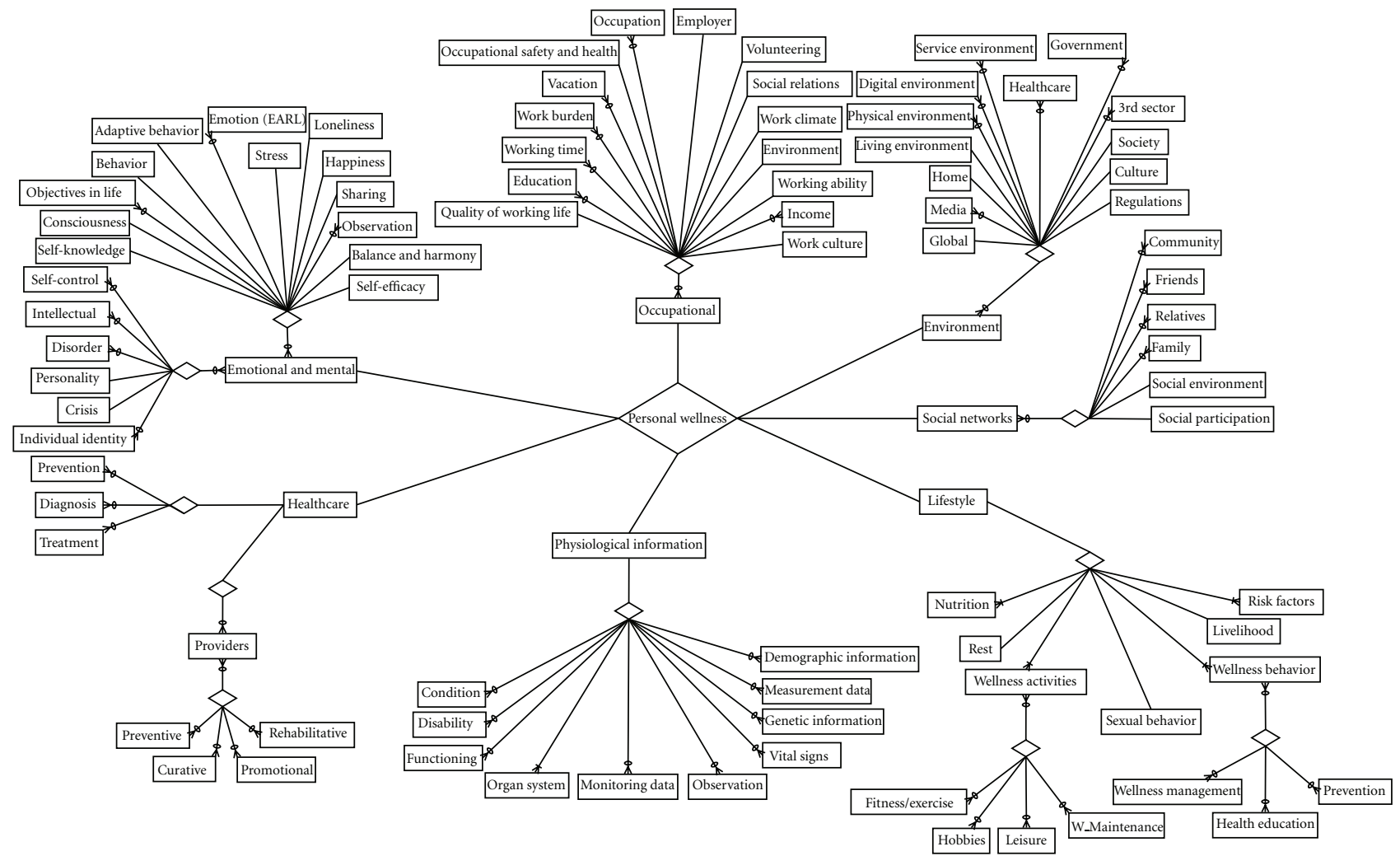

FIGURE 4: The highest level concepts of personal wellness.

and conscious people who are not health professionals or representatives of any health organizations.

The model is based on the focus group research, and we have tried to model the domain and the concepts according to the participants. The guiding principles of the modeling work were how citizens see their personal wellness, what they consider to be the important concepts, how they categorize these concepts, and how they understand the notion of complete, holistic health and wellness. The research process 
was performed iteratively, following the build-evaluate idea of design science research.

We have identified the main components of personal wellness and described related information and its conceptualization. In the model, we have identified the environmental and external contexts related to personal wellness. In our model, we present many important concepts. Based on our categorization of these, we can come to understand the basic relations between concepts, and we can see some isa relationships. There are still some limitations related to the defined concepts in terms of the development of the ontology, as some of the concepts are quite abstract, and their explicit definition can be challenging. Also, there is a great number of relations and relationship types in the domain, and most of the concepts are interconnected.

Hevner et al. [22] have defined seven guidelines that should be followed when performing design science research. The first guideline is to design as an artifact. In our research, we have built artifacts such as mind maps to describe common concepts - the constructs of the domain - and a high-level information model to describe the domain, how it is conceptualized, its scope, and some relationships. The second guideline is problem relevance, which means that the research problem should be relevant and solve some important problem in the constituent community. Pervasive healthcare and a more personalized, complete way of managing personal health and wellness are emerging, yet currently there is no real consensus regarding what personal wellness actually is or its components. Most of the research done on wellness has focused on the measurement or assessment of wellness. Also, the models related to wellness are high-level descriptions with limited conceptual definitions and relationships. Most of the information models and ontologies in the health domain are representations from the health service providers, clinical, or medical points of view. Our research brings new perspectives into the personal wellness domain by defining the citizens' perspective to support the development of a personal wellness ontology and semantic interoperability in the domain. Thus, our research problem is relevant in the personalized health and wellness domain.

The third guideline is about design evaluation. Hevner et al. [22] emphasized that the utility, quality, and efficacy of an artifact must be demonstrated by evaluation, which is a crucial part of the design process. Our design process was iterative, so we built and evaluated the model in various phases, but the evaluation of the model alone still insufficient. The study continues with an assessment phase, in which the developed model is evaluated with respect to its validity and potential impact on citizens' personal health and wellness. The model will be evaluated with use cases and usage scenarios. Design science research, according to the fourth guideline, should clearly contribute in at least one of the following ways: the design artifact, design construction knowledge, or design evaluation knowledge [22]. Our research makes a significant contribution by defining the personal wellness domain, how it is conceptualized, and what the necessary concepts and external factors are.

The fifth guideline emphasizes that the research should be conducted with rigorous methods. As the domain in question was complex, multidimensional, quite abstract, and based on citizens' perspectives, we began with qualitative methods, like focus groups and informal modeling methods. Although, the informal models lacked with formalism we were able to represent the complex and multidimensional domain and the models were rather easy for average citizens with no modeling experience to understand, evaluate, and modify. We had several different focus groups to get more heterogeneous views of personal wellness so that we could tackle the sixth guideline. This guideline emphasizes the importance of an iterative design process and insists that design should be a process of searching such that the design problem becomes more focused and the solution more relevant [22]. The next step in this study is the development of the ontology and context-aware architecture. Thus, the solution will be redefined in a process of searching. The seventh guideline is that the research should be communicated to technology and management audiences. This will be realized in later phases of the project.

\section{Conclusions}

In this research, we have approached complete, holistic health and well-being from the citizens' perspective. We have further refined the concept of personal wellness through empirical methods. Based on an analysis of the literature, a context analysis, and empirical research, we have created our own view of personal wellness. In order to support interoperable, citizen-centered services with upcoming pervasive wellness tools, we have identified the scope of the personal wellness domain. As a result of this research, we have developed a high-level information model that describes the main concepts and some relationships related to personal wellness. With this information model, we have started to develop a context-aware ontology. Our research brings new knowledge to the field of pervasive healthcare by identifying the citizens' perspectives and conceptualizations of personal wellness.

Our model describes the domain of personal wellness in more detail than other models that have been developed in clinical and counseling psychology. Such previous models remain general, while we have gone deeper into the personal wellness domain by defining more concepts, relationships, and properties. Moreover, the focus of our model is different from previous work, as we are addressing the problem from an information system science perspective, and we are using a different kind of representation style that is more suitable in our context. We are focusing on the pervasive healthcare field, and our intentions are to support the development of a personal wellness ontology for lifelong personal wellness management and an architecture model for trusted use of multisource heterogeneous information.

The next phases in our research are an assessment of our model and the development of the personal wellness ontology. The assessment phase focuses on the evaluation of the validity and utility of the model and the identification of the impact the shared use of wellness information has on citizens' wellness management and on how citizens can control and manage the use of their information. In the development of the personal wellness ontology, we have to model the 
contextual aspects that are related to different concepts to support multiuser and multisystem environments with heterogeneous information sources. Furthermore, we have to consider privacy, confidentiality, and data security issues in the ontology, as most of the information is private and personal, and its processing may be regulated by legislation. By modeling such privacy and security aspects, we can ensure that, in the future, pervasive healthcare will allow citizens to control the processing of their information dynamically.

\section{Acknowledgment}

The authors acknowledge the funding of this Trusted eHealth and eWelfare Space (THEWS) research project by the Finnish Academy of Sciences in the MOTIVE Research Programme during 2009-2012.

\section{References}

[1] P. Nykänen, "Requirements for user friendly personal ehealth information systems," in Studies in Health Technology and Informatics, vol. 137, pp. 367-372, IOS Press, 2008.

[2] L. L. Berry and A. M. Mirabito, "Innovative healthcare delivery," Business Horizons, vol. 53, no. 2, pp. 157-169, 2010.

[3] C. Koop, R. Mosher, L. Kun et al., "Future delivery of health care: cybercare," IEEE Engineering in Medicine and Biology Magazine, vol. 27, no. 6, pp. 29-38, 2008.

[4] M. Ohashi, M. Hori, and S. Suzuki, "Citizen-centric ehealthcare management based on pervasive authenticationNew ICT roadmap to active ageing," in 2010 4th International Conference on Pervasive Computing Technologies for Healthcare, Pervasive Health 2010, pp. 1-8, March 2010.

[5] W. Pratt, K. Unruh, A. Civan, and M. Skeels, "Personal health information management," Communications of the ACM, vol. 49, no. 1, pp. 51-55, 2006.

[6] P. Nykänen and A. Seppälä, "Collaborative approach for sustainable citizen-centered health care," in Critical Issues for the Development of Sustainable E-Health Solutions, Healthcare Delivery in the Information Age, N. Wickramasinghe et al., Ed., pp. 115-134, Springer, New York, NY, USA, 2012.

[7] J. S. Larson, "The conceptualization of health," Medical Care Research and Review, vol. 56, no. 2, pp. 123-136, 1999.

[8] World Health Organization, Preamble to the Constitution of the World Health Organization as Adopted by the International Health Conference, World Health Organization, New York, NY, USA, 1948.

[9] S. Mackey, "Towards an ontological theory of wellness: a discussion of conceptual foundations and implications for nursing," Nursing Philosophy, vol. 10, no. 2, pp. 103-112, 2009.

[10] N. Oguz-Duran and E. Tezer, "Wellness and self-esteem among turkish university students," International Journal for the Advancement of Counselling, vol. 31, no. 1, pp. 32-44, 2009.

[11] I. Korhonen and J. E. Bardram, "Introduction to the special section on pervasive healthcare," IEEE Transactions on Information Technology in Biomedicine, vol. 8, no. 3, pp. 229-234, 2004.

[12] B. Arnrich, O. Mayora, J. Bardram, and G. Tröster, "Pervasive healthcare paving the way for a pervasive, user-centered and preventive healthcare model," Methods of Information in Medicine, vol. 49, no. 1, pp. 67-73, 2010.

[13] J. E. Bardram, "Pervasive healthcare as a scientific discipline,"
Methods of Information in Medicine, vol. 47, no. 3, pp. 178-185, 2008.

[14] U. Varshney, "Pervasive healthcare and wireless health monitoring," Mobile Networks and Applications, vol. 12, no. 2-3, pp. 113-127, 2007.

[15] K. Siau and Y. Wang, "Cognitive evaluation of information modeling methods," Information and Software Technology, vol. 49, no. 5, pp. 455-474, 2007.

[16] Y. Wand, D. E. Monarchi, J. Parsons, and C. C. Woo, “Theoretical foundations for conceptual modelling in information systems development," Decision Support Systems, vol. 15, no. 4, pp. 285-304, 1995.

[17] R. Weber, "Conceptual modelling and ontology: possibilities and pitfalls," Journal of Database Management, vol. 14, no. 3, pp. 1-20, 2003.

[18] H. S. Pinto and J. P. Martins, "Ontologies: how can they be built?" Knowledge and Information Systems, vol. 6, no. 4, pp. 441-464, 2004.

[19] H. S. Pinto and J. P. Martins, Ontologies: How Can They Be Built? Springer, New York, NY, USA, 2004.

[20] P. Nykänen, P. Ruotsalainen, B. Blobel, and A. Seppälä, "Research on trusted personal health and wellness information in ubiquitous health information space," in Proceedings of the International Federation for Medical and Biological Engineering (IFMBE '09), pp. 432-435, September 2009.

[21] S. T. March and G. F. Smith, "Design and natural science research on information technology," Decision Support Systems, vol. 15, no. 4, pp. 251-266, 1995.

[22] A. R. Hevner, S. T. March, J. Park, and S. Ram, "Design science in information systems research," MIS Quarterly, vol. 28, no. 1, pp. 75-105, 2004.

[23] D. Zowghi and C. Coulin, "Requirements elicitation: a survey of techniques, approaches, and tools," Engineering and Managing Software Requirements, pp. 19-46, 2005.

[24] J. Kitzinger, "Introducing focus groups," BMJ, vol. 311, no. 7000, pp. 299-302, 1995.

[25] R. A. Powell and H. M. Single, "Focus groups," International Journal for Quality in Health Care, vol. 8, no. 5, pp. 499-504, 1996.

[26] J. E. Myers and T. J. Sweeney, "The indivisible self: an evidencebased model of wellness," Journal of Individual Psychology, vol. 60, no. 3, pp. 234-245, 2004.

[27] C. Soomlek and L. Benedicenti, "Operational wellness model: a wellness model designed for an agent-based wellness visualization system," in 2nd International Conference on eHealth, Telemedicine, and Social Medicine (ETELEMED '10), pp. 4550, February 2010.

[28] T. G. J. C. Kirsten, H. J. L. van der Walt, and C. T. Viljoen, "Health, well-being and wellness: an anthropological ecosystemic approach," Health SA Gesondheid, vol. 14, pp. 1-7, 2009.

[29] T. L. Schuster, M. Dobson, M. Jauregui, and R. H. I. Blanks, "Wellness lifestyles I: a theoretical framework linking wellness, health lifestyles, and complementary and alternative medicine," Journal of Alternative and Complementary Medicine, vol. 10, no. 2, pp. 349-356, 2004.

[30] P. Conrad, "Wellness as virtue: morality and the pursuit of health," Culture, Medicine and Psychiatry, vol. 18, no. 3, pp. 385-401, 1994.

[31] E. W. Sterling, S. A. Von Esenwein, S. Tucker, L. Fricks, and B. G. Druss, "Integrating wellness, recovery, and self-management for mental health consumers," Community Mental Health Journal, vol. 46, no. 2, pp. 130-138, 2010. 
[32] R. A. Kiefer, "An integrative review of the concept of wellbeing," Holistic Nursing Practice, vol. 22, no. 5, pp. 244-252, 2008.

[33] A. Ahtinen, S. Ramiah, J. Blom, and M. Isomursu, "Design of mobile wellness applications: identifying cross-cultural factors," in Proceedings of the 20th Australasian Conference on Computer-Human Interaction: Designing for Habitus and Habitat (OZCHI '08), pp. 164-173, December 2008.

[34] D. A. Els and R. P. De La Rey, "Developing a holistic wellness model," SA Journal of Human Resource Management, no. 2, pp. 46-56, 2006.

[35] T. Sweeney and J. M. Witmer, "Beyond social interest: striving toward optimum health and wellness," Individual Psychology, vol. 47, pp. 527-540, 1991.

[36] C. Saylor, "The circle of health: a health definition model," Journal of Holistic Nursing, vol. 22, no. 2, pp. 97-115, 2004.

[37] A. Seppälä and P. Nykänen, "Contextual analysis and modeling of personal wellness," in Proceedings of the International Conference Knowledge Engineering and Ontology Development (KEOD '11), J. Filipe and J. L. G. Dietz, Eds., pp. 26-29, Paris, France, October 2011.

[38] HUMAINE Emotion Annotation and Representation Language (EARL): Proposalm, 2006, http://emotion-research.net/ projects/humaine/earl/proposal. 

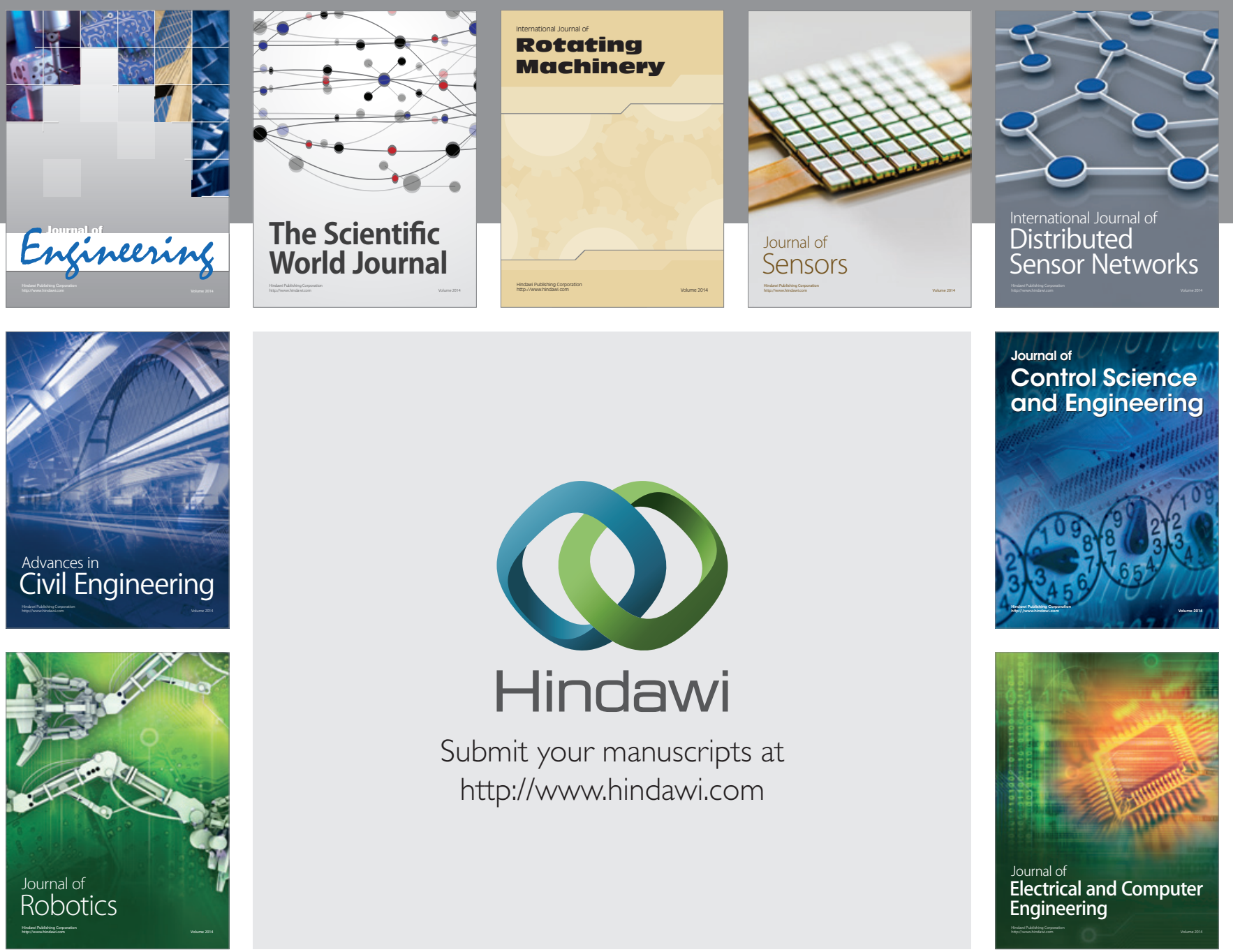

Submit your manuscripts at

http://www.hindawi.com
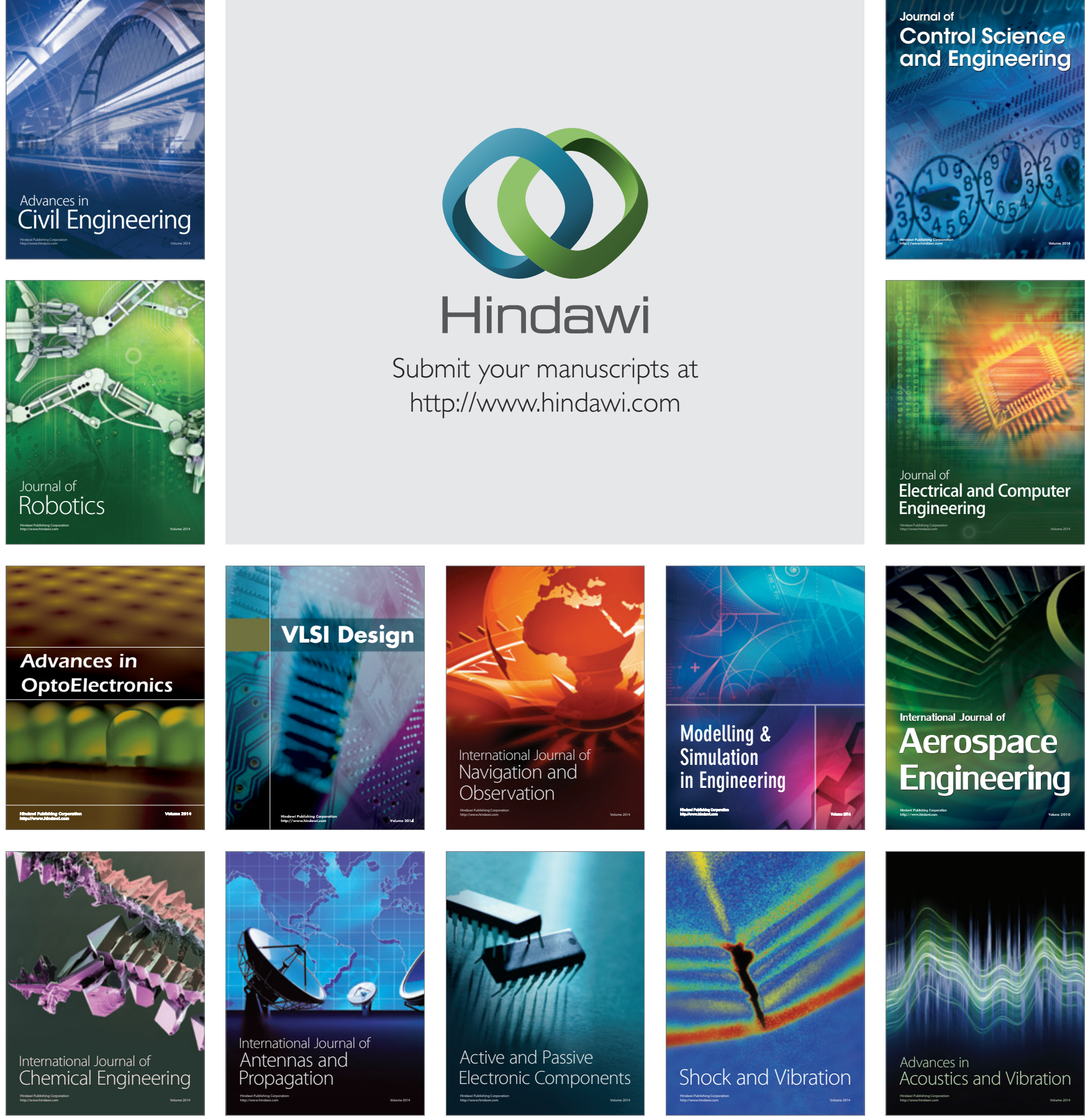